\title{
Differences in Direct Effects of Adrenergic Stimuli on Coronary, Cutaneous, and Muscular Vessels
}

\author{
Alim l. Mark, Francois M. Abboud, Phillip G. Schmm, Donald D. Heistad, and \\ HowARD E. MAYER with the technical assistance of U. JAMEs JohanNSEN \\ From the Cardiovascular Division, Department of Internal Medicine, \\ University of Iowa College of Medicine and The Veterans Administration \\ Hospital, Iowa City, Iowa 52240
}

\begin{abstract}
A в S T R A C T Direct effects of adrenergic stimuli on coronary vessels in dogs were compared with effects on vessels to skin (hind paw) and skeletal muscle (gracilis muscle) after intravenous administration of practolol (2 $\mathrm{mg} / \mathrm{kg}$ ), a selective myocardial beta receptor blocker which minimized indirect effects of myocardial stimulation on coronary vascular resistance. The left circumflex coronary, cranial tibial, and gracilis arteries were perfused separately but simultaneously at constant flow. Perfusion pressures, left ventricular pressure and $\mathrm{dP} /$ $\mathrm{dt}$, and heart rate were recorded. Changes in perfusion pressure to each bed reflected changes in vascular resistance.

The direct constrictor effects of sympathetic nerve stimulation, norepinephrine and phenylephrine on coronary vessels were minimal compared with effects on cutaneous and muscular vessels. Subsequent blockade of vascular beta receptors did not augment the constrictor responses. Angiotensin, a nonadrenergic stimulus, produced striking coronary vasoconstriction which exceeded that in skin and approximated that in muscle. These results suggest that there is a paucity of alpha adrenergic receptors in coronary vessels compared to cutaneous and muscular vessels.

Direct dilator responses to isoproterenol were similar in coronary and cutaneous vessels, but were greater in muscular vessels. Responses to glyceryl trinitrate, a nonadrenergic dilator, also were greater in skeletal muscle. Therefore, differences in effects of isoproterenol on the three beds may reflect differences in reactivity to dilator stimuli rather than differences in the density of beta receptors.
\end{abstract}

This work was presented in part at the 43rd Scientific Sessions of the American Heart Association, Atlantic City, 13 November 1970. A preliminary report has appeared in abstract form (1).

Received for publication 3 May 1971 and in revised form 27 September 1971.
In contrast to norepinephrine, the predominant direct effect of epinephrine on coronary vessels was dilatation mediated through activation of vascular beta receptors. A constrictor effect caused by stimulation of alpha receptors was unmasked by propranolol.

Finally, the order of potency of agonists in stimulating coronary vascular beta receptors and the demonstration of selective beta receptor blockade with practolol suggest that beta receptors in coronary vessels resemble those in peripheral vessels more than those in myocardium.

\section{INTRODUCTION}

Several investigators have reported studies of coronary vascular responses to adrenergic stimuli (2-10), but until recently it has been difficult in the intact beating heart to separate the direct effects of these stimuli on coronary vessels from indirect effects resulting from stimulation of the myocardium. Consequently, there is little available information comparing direct effects of adrenergic stimuli on coronary vessels with effects on vessels in other vascular beds. Recently we studied coronary vascular responses to adrenergic stimuli in the intact beating heart using practolol (11), a new agent which in low doses blocks myocardial but not vascular beta receptors (12). These studies indicated that the coronary vasodilator action of sympathetic nerve stimulation and norepinephrine was indirect and caused by activation of beta receptors in the myocardium. The direct effect of nerve stimulation and norepinephrine on coronary vessels was constriction, but the constrictor responses seemed small compared to those reported with the same stimuli in other vascular beds.

The present experiments were done to compare the direct effects of adrenergic stimuli on coronary vessels with effects on vessels to skin and skeletal muscle, and to identify the mechanisms for differences in coronary responses and responses in skin and skeletal muscle. 
TABLE I

Base Line Observations before and after Practolol ( $2 \mathrm{mg} / \mathrm{kg}$ i.v.)

\begin{tabular}{lcccccc}
\hline & $\begin{array}{l}\text { Coronary } \\
\text { perfusion } \\
\text { pressure }\end{array}$ & $\begin{array}{c}\text { Paw } \\
\text { perfusion } \\
\text { pressure }\end{array}$ & $\begin{array}{c}\text { Gracilis } \\
\text { perfusion } \\
\text { pressure }\end{array}$ & $\begin{array}{c}\text { Left } \\
\text { ventricular } \\
\mathrm{dP} / \mathrm{dt}\end{array}$ & $\begin{array}{c}\text { Left } \\
\text { ventricular } \\
\text { pressure }\end{array}$ & $\begin{array}{c}\text { Heart } \\
\text { rate }\end{array}$ \\
\hline & $\mathrm{mm} \mathrm{Hg}$ & $\mathrm{mm} \mathrm{Hg}$ & $\mathrm{mm} \mathrm{Hg}$ & $\mathrm{mm} \mathrm{Hg} / \mathrm{sec}$ & $\mathrm{mm} \mathrm{Hg}$ & beals/min \\
Before practolol & $87 \pm 7$ (mean $\pm \mathrm{SE})$ & $93 \pm 8$ & $94 \pm 7$ & $3358 \pm 144$ & $162 \pm 7$ & $159 \pm 8$ \\
After practolol & $106 \pm 8$ & $104 \pm 10$ & $107 \pm 9$ & $2770 \pm 162$ & $151 \pm 6$ & $140 \pm 5$ \\
$P$ value & $<0.01$ & $>0.05$ & $<0.05$ & $<0.01$ & $<0.05$ & $<0.01$ \\
(n) & 19 & 19 & 19 & 19 & 19 & 17 \\
\hline
\end{tabular}

\section{METHODS}

Male mongrel dogs weighing 19-28 $\mathrm{kg}$ were anesthetized with chloralose, $50 \mathrm{mg} / \mathrm{kg}$, and urethane, $500 \mathrm{mg} / \mathrm{kg}$. The animals were treated with decamethonium bromide, $0.3 \mathrm{mg} /$ $\mathrm{kg}$, and ventilated artificially with room air and supplemental oxygen at 2-3 liters $/ \mathrm{min}$. In four experiments arterial $\mathrm{pO}_{2}$ averaged $123 \pm 20(\mathrm{sE}) \mathrm{mm} \mathrm{Hg}, \mathrm{pCO}_{2}$ averaged $33 \pm 2 \mathrm{~mm} \mathrm{Hg}$ and $\mathrm{pH}$ averaged $7.38 \pm 0.01$. Through a left thoracotomy, the pericardium was incised and a short segment of the left circumflex coronary artery near its origin was exposed. The cranial tibial artery to the hind paw was exposed near the tarsus and collateral arteries at this level were ligated. In the same hind limb, the gracilis muscle was dissected free from all of its vascular connections with the exception of the gracilis artery and vein. The coronary, tibial, and gracilis arteries then were cannulated and perfused at constant flow with heparinized blood from the femoral artery using perfusion pumps. The cannula from the femoral artery was trifurcated upstream from the pumps to permit separate and simultaneous perfusion of the three arteries. Flows ranged from 48 to $56 \mathrm{ml} / \mathrm{min}$ to the coronary artery, 24 to $32 \mathrm{ml} / \mathrm{min}$ to the hind paw, and 14 to $17 \mathrm{ml} / \mathrm{min}$ to the gracilis muscle. These flows yielded perfusion pressures in the same range as systemic arterial pressure before denervation. With flow constant, changes in perfusion pressure to each bed reflected changes in vascular resistance for that vascular bed. Responses to stimuli were taken as peak changes in perfusion pressure. Perfusion pressures fell abruptly to $10-20 \mathrm{~mm} \mathrm{Hg}$ when the pumps were stopped and there was essentially no back flow of blood from the distal end of the partially transected vessels suggesting that there was little or no collateral flow. In addition to perfusion pressures, we measured aortic and left ventricular pressures, left ventricular $\mathrm{dP} / \mathrm{dt}\left(\mathrm{LV}^{1} \mathrm{dP} /\right.$ $\mathrm{dt}$ ), and heart rate. $\mathrm{LV} \mathrm{dP} / \mathrm{dt}$ was obtained using a No. 7F cardiac catheter, a Statham $\mathrm{P} 23 \mathrm{Db}$ pressure transducer, and an RC differentiating circuit with a time constant of $0.5 \mathrm{msec}$. Beat to beat changes in heart rate were measured using a cardiotachometer.

Drugs used as agonists were 1-norepinephrine bitartrate, phenylephrine hydrochloride, val $^{5}$-angiotensin-11 amide, isoproterenol hydrochloride, glyceryl trinitrate, and epinephrine hydrochloride. Doses of norepinephrine are expressed in terms of base. Fresh solutions were prepared for each experiment using appropriate dilutions of stock solutions in $5 \%$ dextrose in water. The agonists were injected into the perfusion tubing before it trifurcated upstream from the pump so that equal concentrations reached each perfused

${ }^{1}$ Abbreviations used in this paper: LV, left ventricular.

${ }^{2}$ Statham Instruments, Inc., Los Angeles, Calif. bed. The drugs were injected in volumes of $0.1-0.2 \mathrm{ml}$; injections of these volumes of dextrose solution alone had no effect. Other drugs used in the study were practolol ${ }^{3}$ and propranolol, which were given intravenously, and phentolamine, which was administered into the perfusion tubing.

Direct electrical nerve stimulation was accomplished by stimulating the regional sympathetic nerves to each bed separately and consecutively at supramaximal voltage with pulses of $4 \mathrm{msec}$ at variable frequency for $30 \mathrm{sec}$. The left cardiac nerves were sectioned close to the left stellate ganglion and stimulated to obtain coronary responses. The distal ends of the cut sciatic and obturator nerves were stimulated to obtain responses in the hind paw and gracilis muscle, respectively. Supramaximal voltage, which was determined for each nerve in each experiment, was usually $10 \mathrm{v}$ for the cardiac nerves, $15-20 \mathrm{v}$ for the obturator nerve and $20-25 \mathrm{v}$ for the sciatic nerve.

In the first series of experiments these methods were used to compare responses of the three beds to nerve stimulation, phenylephrine, angiotensin, and isoproterenol after intravenous administration of practolol, $2 \mathrm{mg} / \mathrm{kg}$, in eight dogs. Angiotensin was administered to determine the reactivity of the coronary vessels to a nonadrenergic constrictor stimulus. Phenylephrine was given to test responses to a relatively pure alpha receptor stimulating agent. Nerve stimulation was performed first followed by administration of the drugs in random order.

In the second series of experiments, we obtained responses to norepinephrine, epinephrine, angiotensin, isoproterenol, and glyceryltrinitrate after practolol, $2 \mathrm{mg} / \mathrm{kg}$ intravenously, in 11 dogs. Glyceryltrinitrate was used as an internal dilator control to compare the reactivity of the three beds to dilator stimuli. Norepinephrine, epinephrine, and isoproterenol were administered to determine the relative order of potency of these agonists in stimulating coronary vascular beta receptors. The injections were repeated after propranolol, $0.1 \mathrm{mg} / \mathrm{kg}$ intravenously, in seven dogs and after phentolamine, $5 \mathrm{mg}$ given into the perfusion tubing, in three other dogs. Propranolol was given to see if blockade of vascular beta receptors augmented coronary constrictor responses to norepinephrine. Phentolamine was administered to see if blockade of the constrictor effect of norepinephrine unmasked a dilator effect. In eight experiments, responses to isoproterenol were obtained after practolol, $1 \mathrm{mg} / \mathrm{kg}$, after another $1 \mathrm{mg} / \mathrm{kg}$ dose of practolol, and then after propranolol, $0.2 \mathrm{mg} / \mathrm{kg}$ intravenously. This was done to test further the selectivity of beta receptors blockade with practolol. In previous experiments (11) we observed that 1 $\mathrm{mg} / \mathrm{kg}$ of practolol blocked myocardial and reduced slightly

${ }^{3}$ Practolol (AY-21,011) was supplied generously by Ayerst Laboratories, New York. 
TABLE II

Responses after Practolol ( $2 \mathrm{mg} / \mathrm{kg}$ i.v.) in eight Dogs

\begin{tabular}{|c|c|c|c|c|c|c|}
\hline & $\begin{array}{c}\Delta \text { Coronary } \\
\text { perfusion } \\
\text { pressure }\end{array}$ & $\begin{array}{c}\Delta \mathrm{Paw} \\
\text { perfusion } \\
\text { pressure }\end{array}$ & $\begin{array}{l}\Delta \text { Gracilis } \\
\text { perfusion } \\
\text { pressure }\end{array}$ & $\begin{array}{c}\Delta \text { Left } \\
\text { ventricular } \\
\mathrm{dP} / \mathrm{dt}\end{array}$ & $\begin{array}{c}\Delta \text { Left } \\
\text { ventricular } \\
\text { pressure }\end{array}$ & $\begin{array}{l}\Delta \text { Heart } \\
\text { rate }\end{array}$ \\
\hline & $m m \mathrm{Hg}$ & $m m \mathrm{Hg}$ & $m m \mathrm{Hg}$ & $\%$ & $m m \mathrm{Hg}$ & beats/min \\
\hline \multicolumn{7}{|c|}{ Nerve stimulation $(\mathrm{Hz})$} \\
\hline 1 & $4 \pm 3($ mean $\pm \mathrm{SE})$ & $29 \pm 7$ & $33 \pm 8$ & $0 \pm 0$ & $1 \pm 1$ & $0 \pm 0$ \\
\hline 3 & $10 \pm 4$ & $69 \pm 21$ & $86 \pm 21$ & $4 \pm 2$ & $4 \pm 2$ & $0 \pm 0$ \\
\hline 10 & $17 \pm 5$ & $99 \pm 33$ & $120 \pm 29$ & $12 \pm 7$ & $11 \pm 7$ & $2 \pm 2$ \\
\hline \multicolumn{7}{|c|}{ Phenylephrine $(\mu \mathrm{g})$} \\
\hline 6.25 & $5 \pm 1$ & $78 \pm 7$ & $44 \pm 7$ & $2 \pm 2$ & $2 \pm 1$ & $0 \pm 0$ \\
\hline 25.0 & $6 \pm 3$ & $131 \pm 16$ & $83 \pm 8$ & $3 \pm 3$ & $3 \pm 2$ & $0 \pm 0$ \\
\hline \multicolumn{7}{|c|}{ Angiotensin $(\mu \mathrm{g})$} \\
\hline 0.5 & $46 \pm 7$ & $24 \pm 3$ & $50 \pm 7$ & $0 \pm 0$ & $6 \pm 2$ & $0 \pm 0$ \\
\hline 2.0 & $70 \pm 7$ & $37 \pm 8$ & $94 \pm 9$ & $10 \pm 5$ & $15 \pm 4$ & $0 \pm 1$ \\
\hline \multicolumn{7}{|c|}{ Isoproterenol $(\mu \mathrm{g})$} \\
\hline 0.0625 & $-21 \pm 7$ & $-17 \pm 4$ & $-31 \pm 4$ & $0 \pm 0$ & $0 \pm 0$ & $0 \pm 0$ \\
\hline 0.25 & $-27 \pm 7$ & $-27 \pm 3$ & $-59 \pm 5$ & $4 \pm 2$ & $3 \pm 1$ & $0 \pm 0$ \\
\hline
\end{tabular}

coronary responses to isoproterenol. If reduction in the coronary responses after $1 \mathrm{mg} / \mathrm{kg}$ of practolol resulted from partial blockade of coronary vascular beta receptors, rather than from inhibition of the indirect dilator responses caused by myocardial stimulation, then another $1 \mathrm{mg} / \mathrm{kg}$ of practolol would be expected to produce additional blockade of coronary vascular beta receptors and further reduction of the dilator responses to isoproterenol.

In the third series of experiments, the catecholamine concentration of the circumflex coronary, cranial tibial, and gracilis arteries from six anesthetized dogs was measured using the improved fluorometric trihydroxyindole method of Häggendal (13). Segments of the gracilis artery in both hind limbs were excised and pooled as were segments of the cranial tibial artery. The heart was removed and segments of the circumflex coronary and its branches were excised rapidly and pooled. The arteries were quickly stripped of loose connective tissue, blotted, weighed, and frozen in liquid nitrogen. The frozen vessels were pulverized in a stainless steel apparatus which had been cooled to the temperature of liquid nitrogen. The crushed frozen tissue was transferred to an ice-cold Potter-Elvehjem grinding tube and homogenized for $1 \mathrm{~min}$ in $0.4 \mathrm{~N}$ perchloric acid. Following centrifugation $(9000 \mathrm{~g})$ at $0^{\circ} \mathrm{C}$ for $15 \mathrm{~min}$, the precipitate was extracted with $0.4 \mathrm{~N} \mathrm{HClO}_{4}$ and recentrifuged. The supernatants were combined. The catecholamines were extracted by the aluminum-oxide adsorption process of Anton and Sayre (14). The eluted catecholamines were analyzed using a modification of the trihydroxyindole procedure of Häggendal (13). Faded tissue blanks and a series of standards were routinely analyzed with each sample. Standards were prepared from pure 1-norepinephrine $\mathrm{HCl}^{4}{ }^{4}$ Fluorescence was measured in a Perkin-Elmer MPF-2A spectrophotometer ${ }^{5}$ equipped with a high sensitivity cell holder. Linear curves were obtained with standards ranging

\footnotetext{
${ }^{4}$ Sigma Chemical Co., St. Louis, Mo.

${ }^{5}$ Perkin-Elmer Corp., Boller \& Chivens Div., South Pasadena, Calif.
}

from 0 to $50 \mathrm{ng}$ of norepinephrine. At high sensitivity settings $10 \mathrm{ng}$ of norepinephrine gave full scale readings. The data has not been corrected for losses in the purification procedure.

Statistical comparisons were performed using the $t$ test for paired data.

\section{RESULTS}

Effects of practolol on base line observations (Table $I$, Figs. 1 and 2). Coronary and gracilis perfusion pressures increased after intravenous administration of practolol while heart rate and left ventricular pressure and $\mathrm{dP} / \mathrm{dt}$ decreased. Perfusion pressure in the paw increased slightly, but not significantly, after practolol.

Responses after practolol (Tables II and III, Figs. 1-3). Nerve stimulation, norepinephrine and phenylephrine resulted in small increases in coronary perfusion pressure compared with large increases in perfusion pressure in the paw and gracilis muscle. In contrast, angiotensin produced large coronary constrictor responses which were greater than the responses in the paw and only slightly less than those in the gracilis muscle. Epinephrine produced coronary dilatation, but caused vasoconstriction in the paw and gracilis muscle which resembled that seen with norepinephrine and

\footnotetext{
${ }^{6}$ In six fluorometric determinations the average recovery of norepinephrine from the alumina extraction process was $76.3 \pm 3.6 \%$ (SEM). In one additional determination using tritiated norepinephrine $\left(1-\mathrm{NE}-7-\mathrm{H}^{3}\right) \quad 93.2 \%$ of the label was adsorbed by the alumina. Successive washes of the alumina removed increasing amounts of the label, up to $6.3 \%$ with the fourth wash. Recovery of $1-\mathrm{NE}-7-\mathrm{H}^{3}$ in the alumina extract was $73.1 \%$.
} 
TABLE III

Responses after Practolol ( $2 \mathrm{mg} / \mathrm{kg}$ i.v.) in Eleven Other Dogs

\begin{tabular}{|c|c|c|c|c|c|c|}
\hline & $\begin{array}{c}\Delta \text { Coronary } \\
\text { perfusion } \\
\text { pressure } \\
m m \mathrm{Hg}\end{array}$ & $\begin{array}{c}\Delta \text { Paw } \\
\text { perfusion } \\
\text { pressure } \\
m m \mathrm{Hg}\end{array}$ & $\begin{array}{c}\Delta \text { Gracilis } \\
\text { perfusion } \\
\text { pressure } \\
m m ~ H g\end{array}$ & $\begin{array}{c}\Delta \text { Left } \\
\text { ventricular } \\
\mathrm{dP} / \mathrm{dt} \\
\%\end{array}$ & $\begin{array}{c}\Delta \text { Left } \\
\text { ventricular } \\
\text { pressure } \\
m m ~ H g\end{array}$ & $\begin{array}{c}\Delta \text { Heart } \\
\text { rate } \\
\text { beats } / \text { min }\end{array}$ \\
\hline \multicolumn{7}{|c|}{ Norepinephrine $(\mu \mathrm{g})$} \\
\hline $\begin{array}{l}1 \\
4\end{array}$ & $\begin{array}{l}12 \pm 4(\text { mean } \pm \mathrm{SE}) \\
12 \pm 5\end{array}$ & $\begin{array}{r}66 \pm 7 \\
121 \pm 9\end{array}$ & $\begin{array}{l}62 \pm 8 \\
86 \pm 10\end{array}$ & $\begin{array}{r}7 \pm 3 \\
24 \pm 6\end{array}$ & $\begin{array}{r}4 \pm 1 \\
14 \pm 2\end{array}$ & $\begin{array}{l}0 \pm 0 \\
3 \pm 4\end{array}$ \\
\hline \multicolumn{7}{|c|}{ Epinephrine $(\mu \mathrm{g})$} \\
\hline $\begin{array}{l}1 \\
4\end{array}$ & $\begin{array}{r}-9 \pm 3 \\
-15 \pm 5\end{array}$ & $\begin{aligned} 65 & \pm 8 \\
123 & \pm 11\end{aligned}$ & $\begin{array}{l}48 \pm 7 \\
79 \pm 15\end{array}$ & $\begin{aligned} 2 & \pm 1 \\
10 & \pm 3\end{aligned}$ & $\begin{array}{l}1 \pm 1 \\
3 \pm 2\end{array}$ & $\begin{array}{r}0 \pm 0 \\
-1 \pm 1\end{array}$ \\
\hline \multicolumn{7}{|c|}{ Angiotensin $(\mu \mathrm{g})$} \\
\hline $\begin{array}{l}0.625 \\
2.5\end{array}$ & $\begin{array}{l}33 \pm 5 \\
45 \pm 7\end{array}$ & $\begin{array}{l}20 \pm 7 \\
35 \pm 5\end{array}$ & $\begin{array}{l}46 \pm 17 \\
59 \pm 15\end{array}$ & $\begin{array}{l}3 \pm 3 \\
2 \pm 2\end{array}$ & $\begin{array}{l}0 \pm 2 \\
7 \pm 3\end{array}$ & $\begin{array}{r}0 \pm 0 \\
-1 \pm 1\end{array}$ \\
\hline \multicolumn{7}{|c|}{ Isoproterenol $(\mu \mathrm{g})$} \\
\hline $\begin{array}{l}0.25 \\
1.0\end{array}$ & $\begin{array}{l}-17 \pm 3 \\
-21 \pm 4\end{array}$ & $\begin{array}{l}-13 \pm 1 \\
-22 \pm 3\end{array}$ & $\begin{array}{l}-24 \pm 4 \\
-39 \pm 6\end{array}$ & $\begin{array}{r}3 \pm 2 \\
14 \pm 3\end{array}$ & $\begin{array}{l}1 \pm 1 \\
5 \pm 2\end{array}$ & $\begin{array}{l}-1 \pm 1 \\
-1 \pm 2\end{array}$ \\
\hline \multicolumn{7}{|c|}{ Glyceryl trinitrate $(\mu \mathrm{g})$} \\
\hline $\begin{array}{r}4 \\
16\end{array}$ & $\begin{array}{l}-19 \pm 4 \\
-28 \pm 5\end{array}$ & $\begin{array}{l}-21 \pm 3 \\
-25 \pm 4\end{array}$ & $\begin{array}{l}-26 \pm 6 \\
-37 \pm 6\end{array}$ & $\begin{array}{l}0 \pm 0 \\
0 \pm 0\end{array}$ & $\begin{array}{l}0 \pm 0 \\
0 \pm 0\end{array}$ & $\begin{array}{l}0 \pm 0 \\
0 \pm 0\end{array}$ \\
\hline
\end{tabular}

phenylephrine. Isoproterenol and glyceryl trinitrate produced dilatation in all three vascular beds. Responses to these various stimuli after practolol occurred without significant changes in heart rate and left ventricular

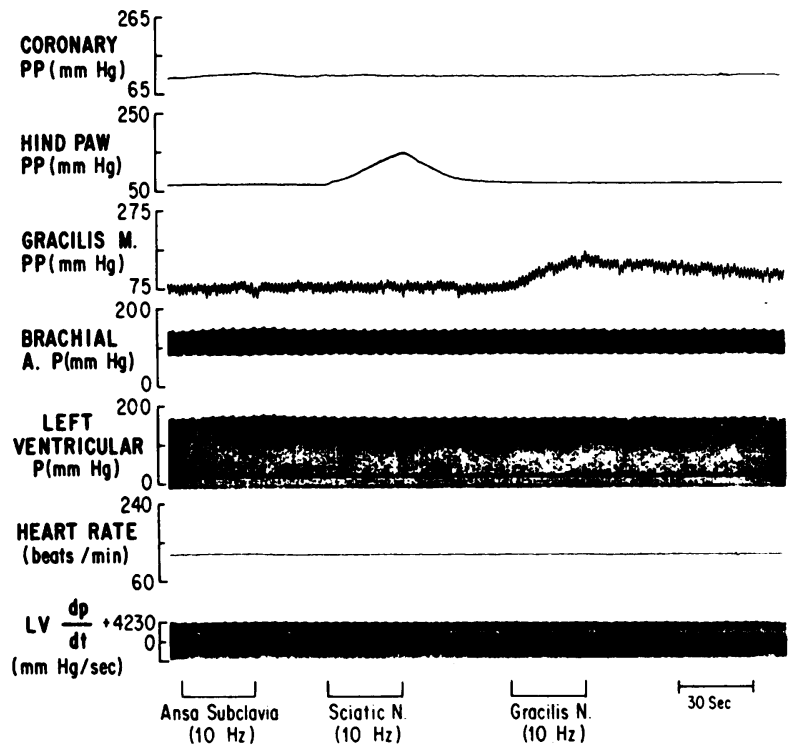

Figure 1 Responses to direct electrical sympathetic nerve stimulation after practolol, $2 \mathrm{mg} / \mathrm{kg}$ intravenously, in one of these experiments. (Exp. No. $129 \mathrm{VAH}$ ) pressure and $\mathrm{dP} / \mathrm{dt}$, with the exception of increases in $\mathrm{dP} / \mathrm{dt}$ and pressure with the high dose of norepinephrine and smaller but significant increases in $\mathrm{dP} / \mathrm{dt}$ with the high dose of isoproterenol and angiotensin $(P<0.05$, Table III).

Effects of propranolol after practolol (Table IV). Propranolol reduced or abolished dilator responses to

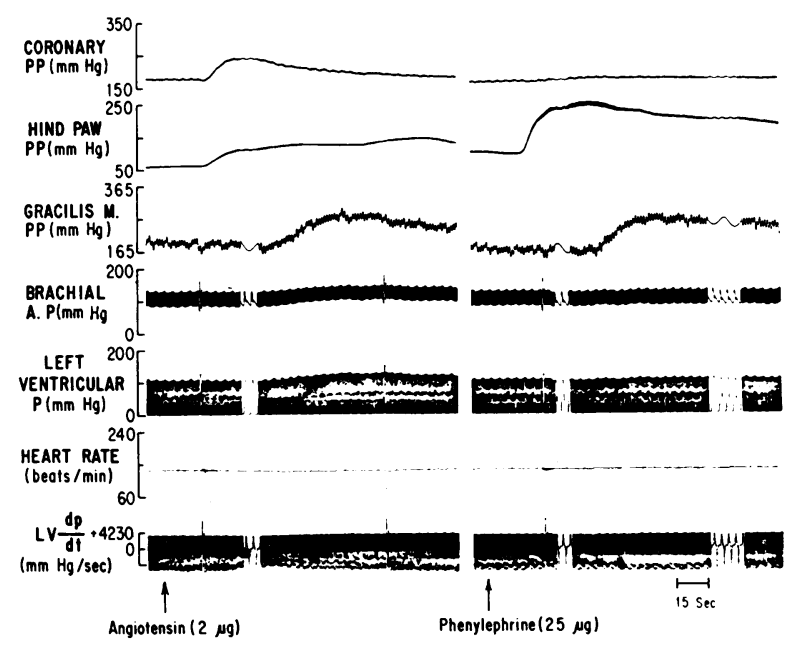

Figure 2 Segments of record showing responses to angiotensin and phenylephrine after practolol, $2 \mathrm{mg} / \mathrm{kg}$ intravenously. (Exp. No. 131 VAH) 
TABLE IV

Comparison of Responses after Practolol (2 mg/kg i.v.) with Responses after Practolol plus Propranolol $(0.1 \mathrm{mg} / \mathrm{kg})$ in Seven Dogs (mean $\pm \mathrm{SE})$

\begin{tabular}{|c|c|c|c|c|c|c|c|c|}
\hline \multirow[b]{3}{*}{ Epinephrine, $\mu \mathrm{g}$} & \multicolumn{2}{|c|}{$\begin{array}{l}\Delta \text { Coronary } \\
\text { perfusion } \\
\text { pressure }\end{array}$} & \multicolumn{2}{|c|}{$\begin{array}{c}\Delta \text { Paw } \\
\text { perfusion } \\
\text { pressure }\end{array}$} & \multicolumn{2}{|c|}{$\begin{array}{l}\Delta \text { Gracilis } \\
\text { perfusion } \\
\text { pressure }\end{array}$} & \multicolumn{2}{|c|}{$\begin{array}{c}\Delta \text { Left } \\
\text { ventricular } \\
\mathrm{dP} / \mathrm{dt}\end{array}$} \\
\hline & \multicolumn{2}{|c|}{$m m \mathrm{Hg}$} & \multicolumn{2}{|c|}{$m m \mathrm{Hg}$} & \multicolumn{2}{|c|}{$m m \mathrm{Hg}$} & \multicolumn{2}{|r|}{$\%$} \\
\hline & 1 & 4 & 1 & 4 & 1 & 4 & 1 & 4 \\
\hline $\begin{array}{l}\text { After practolol } \\
\text { After practolol }\end{array}$ & $-11 \pm 4$ & $-16 \pm 7$ & $50 \pm 5$ & $104 \pm 10$ & $31 \pm 12$ & $60 \pm 20$ & $3 \pm 2$ & $6 \pm 3$ \\
\hline $\begin{array}{l}\text { and propranolol } \\
P \text { value }\end{array}$ & $\begin{array}{l}10 \pm 5 \\
<0.01\end{array}$ & $\begin{array}{l}8 \pm 5 \\
<0.05\end{array}$ & $\begin{array}{l}68 \pm 10 \\
<0.05\end{array}$ & $\begin{array}{l}132 \pm 15 \\
<0.05\end{array}$ & $\begin{array}{l}49 \pm 17 \\
>0.05\end{array}$ & $\begin{array}{l}84 \pm 18 \\
>0.05\end{array}$ & $\begin{array}{l}0 \pm 0 \\
>0.05\end{array}$ & $\begin{array}{l}10 \pm 5 \\
>0.05\end{array}$ \\
\hline Norepinephrine, $\mu \mathrm{g}$ & 1 & 4 & 1 & 4 & 1 & 4 & 1 & 4 \\
\hline $\begin{array}{l}\text { After practolol } \\
\text { After practolol }\end{array}$ & $9 \pm 6$ & $11 \pm 7$ & $55 \pm 6$ & $114 \pm 11$ & $53 \pm 15$ & $86 \pm 15$ & $6 \pm 3$ & $22 \pm 9$ \\
\hline $\begin{array}{l}\text { and propranolol } \\
P \text { value }\end{array}$ & $\begin{array}{c}13 \pm 9 \\
>0.05\end{array}$ & $\begin{array}{c}9 \pm 11 \\
>0.05\end{array}$ & $\begin{array}{l}63 \pm 12 \\
>0.05\end{array}$ & $\begin{array}{c}120 \pm 14 \\
>0.05\end{array}$ & $\begin{array}{l}40 \pm 6 \\
>0.05\end{array}$ & $\begin{array}{l}58 \pm 12 \\
>0.05\end{array}$ & $\begin{array}{l}8 \pm 5 \\
>0.05\end{array}$ & $\begin{array}{r}23 \pm 5 \\
>0.05\end{array}$ \\
\hline Isoproterenol, $\boldsymbol{\mu g}$ & 0.25 & 1 & 0.25 & 1 & 0.25 & 1 & 0.25 & 1 \\
\hline $\begin{array}{l}\text { After practolol } \\
\text { After practolol }\end{array}$ & $-17 \pm 5$ & $-22 \pm 5$ & $-14 \pm 2$ & $-24 \pm 3$ & $-26 \pm 6$ & $-40 \pm 10$ & $1 \pm 1$ & $12 \pm 4$ \\
\hline $\begin{array}{l}\text { and propranolol } \\
P \text { value }\end{array}$ & $\begin{array}{l}-3 \pm 2 \\
<0.05\end{array}$ & $\begin{array}{c}-15 \pm 6 \\
<0.05\end{array}$ & $\begin{array}{c}-1 \pm 1 \\
<0.01\end{array}$ & $\begin{array}{l}-7 \pm 2 \\
<0.01\end{array}$ & $\begin{array}{l}-4 \pm 1 \\
<0.05\end{array}$ & $\begin{array}{c}-11 \pm 2 \\
<0.05\end{array}$ & $\begin{array}{c}1 \pm 1 \\
>0.05\end{array}$ & $\begin{array}{l}24 \pm 5 \\
<0.05\end{array}$ \\
\hline
\end{tabular}

isoproterenol and reversed coronary responses to epinephrine from dilator to constrictor. After propranolol, constrictor responses to epinephrine were increased significantly in the paw and slightly, but not significantly, in the gracilis muscle. Constrictor responses to norepinephrine were not augmented by propranolol. Responses to glyceryl trinitrate, used as an internal dilator control, were not reduced.

Effects of phentolamine after practolol (Table $V$ ). Phentolamine reduced, but did not reverse, constrictor responses to norepinephrine. Constrictor responses to epinephrine were reduced in the paw and reversed in the gracilis muscle; coronary dilator responses to epinephrine were not altered.

Catecholamine concentration of vessels. The catecholamine concentration averaged $1.86 \pm 0.49 \mu \mathrm{g} / \mathrm{g}$ in segments of cranial tibial arteries, $0.82 \pm 0.22 \mu \mathrm{g} / \mathrm{g}$ in circumflex arteries and $0.19 \pm 0.00 \mu \mathrm{g} / \mathrm{g}$ in gracilis arteries. The concentration in cranial tibial and coronary vessels did not differ significantly $(P>0.05)$, but the concentration of both exceeded that in the gracilis arteries $(P<0.05)$.

TABLE V

Comparison of Responses after Practolol $(2 \mathrm{mg} / \mathrm{kg}$ i.v.) with Responses after Practolol plus Phentolamine (5 mg i.a.) in Three Dogs (mean $\pm \mathrm{SE}$ )

\begin{tabular}{|c|c|c|c|c|c|c|c|c|}
\hline \multirow{7}{*}{$\begin{array}{l}\text { Epinephrine, } \mu \mathrm{g} \\
\text { After practolol } \\
\text { After practolol } \\
\text { and phentolamine } \\
P \text { value }\end{array}$} & \multirow{2}{*}{\multicolumn{2}{|c|}{$\begin{array}{c}\begin{array}{c}\Delta \text { Coronary } \\
\text { perfusion } \\
\text { pressure }\end{array} \\
\mathbf{m m ~} \mathbf{H g}\end{array}$}} & \multicolumn{2}{|c|}{$\begin{array}{c}\Delta \mathrm{Paw} \\
\text { perfusion } \\
\text { pressure }\end{array}$} & \multicolumn{2}{|c|}{$\begin{array}{l}\Delta \text { Gracilis } \\
\text { perfusion } \\
\text { pressure }\end{array}$} & \multicolumn{2}{|c|}{$\begin{array}{c}\Delta \text { Left } \\
\text { ventricular } \\
\mathrm{dP} / \mathrm{dt}\end{array}$} \\
\hline & & & \multicolumn{2}{|c|}{$m m \mathrm{Hg}$} & \multicolumn{2}{|c|}{$m m \mathrm{Hg}$} & \multicolumn{2}{|c|}{$\%$} \\
\hline & 1 & 4 & 1 & 4 & 1 & 4 & 1 & 3 \\
\hline & $-8 \pm 7$ & $-18 \pm 3$ & $100 \pm 6$ & $165 \pm 14$ & $67 \pm 12$ & $117 \pm 12$ & $2 \pm 2$ & $18 \pm 3$ \\
\hline & & & & & & & & \\
\hline & $-13 \pm 3$ & $-20 \pm 3$ & $8 \pm 3$ & $13 \pm 7$ & $-13 \pm 8$ & $-12 \pm 7$ & $2 \pm 2$ & $15 \pm 1$ \\
\hline & $>0.05$ & $>0.05$ & $<0.01$ & $<0.05$ & $<0.05$ & $<0.01$ & $>0.05$ & $>0.05$ \\
\hline Norepinephrine, $\mu \mathrm{g}$ & 1 & 4 & 1 & 4 & 1 & 4 & 1 & 4 \\
\hline After practolol & $18 \pm 7$ & $18 \pm 3$ & $93 \pm 3$ & $142 \pm 15$ & $65 \pm 12$ & $88 \pm 6$ & $13 \pm 3$ & $32 \pm 9$ \\
\hline After practolol & & & & & & & & \\
\hline and phentolamine & $2 \pm 2$ & $2 \pm 4$ & $8 \pm 6$ & $25 \pm 10$ & $22 \pm 4$ & $45 \pm 5$ & $18 \pm 5$ & $33 \pm 6$ \\
\hline$P$ value & $>0.05$ & $>0.05$ & $<0.01$ & $<0.01$ & $<0.05$ & $<0.05$ & $>0.05$ & $>0.05$ \\
\hline
\end{tabular}


TABLE VI

Responses to Isoproterenol after Two Doses of Practolol $(1 \mathrm{mg} / \mathrm{kg}$ and

\begin{tabular}{|c|c|c|c|c|c|c|}
\hline & \multicolumn{3}{|c|}{$\Delta$ Coronary perfusion pressure } & \multicolumn{3}{|c|}{$\Delta$ Paw perfusion pressure } \\
\hline & \multicolumn{3}{|c|}{$m m \mathbf{H g}$} & \multicolumn{3}{|c|}{$m m \mathrm{Hg}$} \\
\hline$\mu \mathrm{g}$ & 0.0625 & 0.25 & 1.0 & 0.0625 & 0.25 & 1.0 \\
\hline Before practolol & $\begin{array}{l}-28 \\
\pm 14\end{array}$ & $\begin{array}{r}-30 \\
\pm 8\end{array}$ & $\begin{array}{r}-33 \\
\pm 9\end{array}$ & $\begin{array}{r}-28 \\
\pm 7\end{array}$ & $\begin{array}{r}-25 \\
\pm 7\end{array}$ & $\begin{array}{r}-32 \\
\pm 7\end{array}$ \\
\hline After practolol, $1 \mathrm{mg} / \mathrm{kg}$ & $\begin{array}{r}-28 \\
\pm 5\end{array}$ & $\begin{array}{r}-28 \\
\pm 4\end{array}$ & $\begin{array}{r}-31 \\
\pm 4\end{array}$ & $\begin{array}{r}-24 \\
\pm 4\end{array}$ & $\begin{array}{r}-27 \\
\pm 7\end{array}$ & $\begin{array}{r}-35 \\
\pm 8\end{array}$ \\
\hline $\begin{array}{l}P \text { value (Before vs. after } \\
\text { practolol, } 1 \mathrm{mg} / \mathrm{kg} \text { ) }\end{array}$ & $>0.05$ & $>0.05$ & $>0.05$ & $>0.05$ & $>0.05$ & $>0.05$ \\
\hline After practolol, $2 \mathrm{mg} / \mathrm{kg}$ & $\begin{array}{r}-29 \\
\pm 4\end{array}$ & $\begin{array}{r}-25 \\
\pm 4\end{array}$ & $\begin{array}{r}-29 \\
\pm 4\end{array}$ & $\begin{array}{r}-23 \\
\pm 4\end{array}$ & $\begin{array}{r}-20 \\
\pm 5\end{array}$ & $\begin{array}{r}-31 \\
\pm 6\end{array}$ \\
\hline $\begin{array}{l}P \text { value (After practolol } 1 \mathrm{mg} / \mathrm{kg} \text { vs. after } \\
\text { practolol, } 2 \mathrm{mg} / \mathrm{kg} \text { ) }\end{array}$ & $>0.05$ & $>0.05$ & $>0.05$ & $>0.05$ & $<0.05$ & $>0.05$ \\
\hline After propranolol, $0.2 \mathrm{mg} / \mathrm{kg}$ & $\begin{array}{r}0 \\
\pm 0\end{array}$ & $\begin{array}{l}-4 \\
\pm 2\end{array}$ & $\begin{array}{l}-3 \\
\pm 3\end{array}$ & $\begin{array}{r}0 \\
\pm 0\end{array}$ & $\begin{array}{r}0 \\
\pm 0\end{array}$ & $\begin{array}{l}-4 \\
\pm 1\end{array}$ \\
\hline $\begin{array}{l}P \text { value (After practolol, } 2 \mathrm{mg} / \mathrm{kg} \text { vs. after } \\
\text { propranolol) }\end{array}$ & $<0.01$ & $<0.05$ & $<0.01$ & $<0.05$ & $<0.05$ & $<0.05$ \\
\hline
\end{tabular}

* Entries represent mean $\pm \mathrm{SE}$ of responses in four dogs for $0.0625 \mu \mathrm{g}$ isoproterenol and in eight dogs for 0.25 and 1.0 $\mu \mathrm{g}$ isoporterenol.

Responses to isoproterenol after two doses of practolol (Table VI). Practolol in doses of $1 \mathrm{mg} / \mathrm{kg}$ and 2 $\mathrm{mg} / \mathrm{kg}$ reduced or abolished myocardial responses to isoproterenol, but did not reduce vasodilator responses. Subsequent administration of propranolol blocked the vasodilator responses without producing further significant inhibition of myocardial responses.

\section{DISCUSSION}

These experiments indicate that the direct constrictor effects of adrenergic stimuli, such as sympathetic nerve stimulation and norepinephrine, on coronary vessels are minimal compared with effects on cutaneous and muscular vessels and suggest that this results from a paucity of alpha adrenergic receptors in coronary vessels compared with vessels to skin and skeletal muscle. We considered other reasons for the small constrictor responses to adrenergic stimuli in coronary vessels. First, since considerable coronary constriction occurred with angiotensin, a nonadrenergic stimulus, the absence of pronounced constrictor effects of adrenergic stimuli on coronary vessels cannot be explained by unresponsiveness of these vessels to all constrictor stimuli. Second, we considered the possibility that the coronary constrictor responses to nerve stimulation and norepinephrine were small because the constrictor effect was opposed by a dilator action resulting from activation of beta receptors in coronary vessels or from residual stimulation of myo- cardial beta receptors after practolol. This is improbable because phenylephrine, a relatively pure alpha receptor stimulating agent (15), also produced only slight coronary constriction and because propranolol in a dose which reduced or abolished responses to isoproterenol did not augment the constriction with norepinephrine. Third, removal of norepinephrine from receptor sites by uptake into sympathetic nerve endings might be greater or faster in coronary vessels than in vessels to skin or skeletal muscle. Since the constrictor effect of phenylephrine, which is not taken up rapidly into sympathetic nerve endings (15), on coronary vessels was minimal compared with effects on cutaneous and muscular vessels, it is unlikely that differences in constrictor effects of adrenergic stimuli on the three beds result from differences in uptake into nerve endings. We conclude, therefore, that the differences in the direct constrictor effects of adrenergic stimuli on coronary, cutaneous, and muscular vessels result from a paucity of alpha receptors in coronary vessels compared with vessels in the other two beds.

Direct dilator responses to the beta receptor stimulating agent, isoproterenol, in the coronary circulation approximated those in the paw, but were less than those in the gracilis muscle. It is difficult to draw conclusions concerning the relative density of beta receptors in the three vascular beds from these observations, because responses to glyceryl trinitrate, used as an internal dilator 
$2 \mathrm{mg} / \mathrm{kg}, i . v$.$) and after Practolol plus Propranolol (0.2 \mathrm{mg} / \mathrm{kg}, i . v .)^{*}$

\begin{tabular}{|c|c|c|c|c|c|c|c|c|c|c|c|}
\hline \multicolumn{3}{|c|}{$\Delta$ Muscle perfusion pressure } & \multicolumn{3}{|c|}{$\Delta$ Left ventricular $\mathrm{dP} / \mathrm{dt}$} & \multicolumn{3}{|c|}{$\Delta$ Left ventricular pressure } & \multicolumn{3}{|c|}{$\Delta$ Heart rate } \\
\hline \multicolumn{3}{|c|}{$m m \mathrm{Hg}$} & \multicolumn{3}{|c|}{$\%$} & \multicolumn{3}{|c|}{$m m \mathrm{Hg}$} & \multicolumn{3}{|c|}{ beats/min } \\
\hline 0.0625 & 0.25 & 1.0 & 0.0625 & 0.25 & 1.0 & 0.0625 & 0.25 & 1.0 & 0.0625 & 0.25 & 1.0 \\
\hline-16 & -16 & -30 & 41 & 47 & 61 & 21 & 26 & 31 & 10 & 20 & 23 \\
\hline \pm 7 & \pm 3 & \pm 6 & \pm 10 & \pm 6 & \pm 10 & \pm 6 & \pm 5 & \pm 4 & \pm 3 & \pm 9 & \pm 10 \\
\hline-21 & -23 & -34 & 3 & 10 & 24 & 3 & 5 & 14 & 0 & 0 & 1 \\
\hline \pm 8 & \pm 4 & \pm 7 & \pm 3 & \pm 3 & \pm 10 & \pm 3 & \pm 2 & \pm 3 & \pm 0 & \pm 0 & \pm 2 \\
\hline$>0.05$ & $>0.05$ & $>0.05$ & $<0.05$ & $<0.01$ & $<0.01$ & $<0.05$ & $<0.01$ & $<0.01$ & $<0.05$ & $>0.05$ & $>0.05$ \\
\hline-26 & -24 & -32 & 0 & 6 & 19 & 0 & 0 & 6 & 0 & 0 & 3 \\
\hline \pm 9 & \pm 6 & \pm 6 & \pm 0 & \pm 4 & \pm 6 & \pm 0 & \pm 1 & \pm 2 & \pm 0 & \pm 0 & \pm 3 \\
\hline$>0.05$ & $>0.05$ & $>0.05$ & $>0.05$ & $>0.05$ & $>0.05$ & $>0.05$ & $<0.05$ & $>0.05$ & $>0.05$ & $>0.05$ & $>0.05$ \\
\hline 0 & -4 & -5 & 1 & 2 & 3 & 1 & 1 & 1 & 0 & 0 & 0 \\
\hline \pm 0 & \pm 2 & \pm 3 & \pm 1 & \pm 2 & \pm 3 & \pm 1 & \pm 1 & \pm 1 & \pm 0 & \pm 0 & \pm 0 \\
\hline$>0.05$ & $>0.05$ & $>0.05$ & $>0.05$ & $>0.05$ & $>0.05$ & $>0.05$ & $>0.05$ & $>0.05$ & $>0.05$ & $>0.05$ & $>0.05$ \\
\hline
\end{tabular}

control, also were larger in the gracilis muscle than in the paw and coronary. Differences in effects of isoproterenol may reflect differences in reactivity of the three beds to dilator stimuli rather than differences in density of beta receptors.

An unexpected finding in this study was the difference in the direct effects of epinephrine and norepinephrine on coronary vessels. Previous studies in fibrillating or potassium-arrested hearts suggested that the direct coronary effect of both norepinephrine and epinephrine is constriction $(4,5)$. In contrast, in studies of isolated strips of coronary vessels from dogs, Zuberbuhler and Bohr found that epinephrine and norepinephrine caused relaxation of strips from small coronary vessels (9). Norepinephrine was much more potent than epinephrine. On strips from large coronary arteries the two agents produced either relaxation or contraction, with norepinephrine more potent as a dilator and epinephrine more potent as a constrictor (9). In the present experiments, the direct effect of epinephrine was coronary vasodilatation; the direct effect of norepinephrine was vasoconstriction. Although the dilator responses to epinephrine after practolol occasionally were accompanied by small increases in left ventricular $\mathrm{dP} / \mathrm{dt}$, similar responses often occurred without changes in $\mathrm{dP} / \mathrm{dt}$, heart rate, and left ventricular pressure (Fig. 3). In addition, after administration of practolol residual increases in $\mathrm{dP} / \mathrm{dt}$ with norepinephrine were greater than with epinephrine, but vasodilatation was not seen with norepinephrine. Administration of propranolol after practolol reversed coronary responses to epinephrine from dilatation to constriction, but did not augment the constriction with norepinephrine. These observations indicate that in these experiments the predominant direct effect of epinephrine on coronary vessels was dilatation mediated through stimulation of vascular beta receptors; a constrictor effect caused by activation of alpha receptors was unmasked by propranolol. The direct effect of norepinephrine was constriction mediated through alpha receptors; vascular beta receptors did not play an important role in responses to norepinephrine.

The catecholamine concentration of coronary arteries exceeded that of gracilis arteries and did not differ significantly from the concentration in cranial tibial arteries, although it tended to be less in coronary vessels. The high concentration in coronary vessels was surprising in view of minimal responsiveness of these vessels to sympathetic nerve stimulation, but the results are consistent with a recent preliminary report by King, Angelakos, and Uzgiris that histochemical techniques reveal dense adrenergic innervation in all coronary vessels (16). The role of the dense adrenergic innervation in control of the coronary circulation remains obscure, since these vessels appear to contain a sparse population of alpha receptors and since vascular beta receptors apparently do not play an important role in 


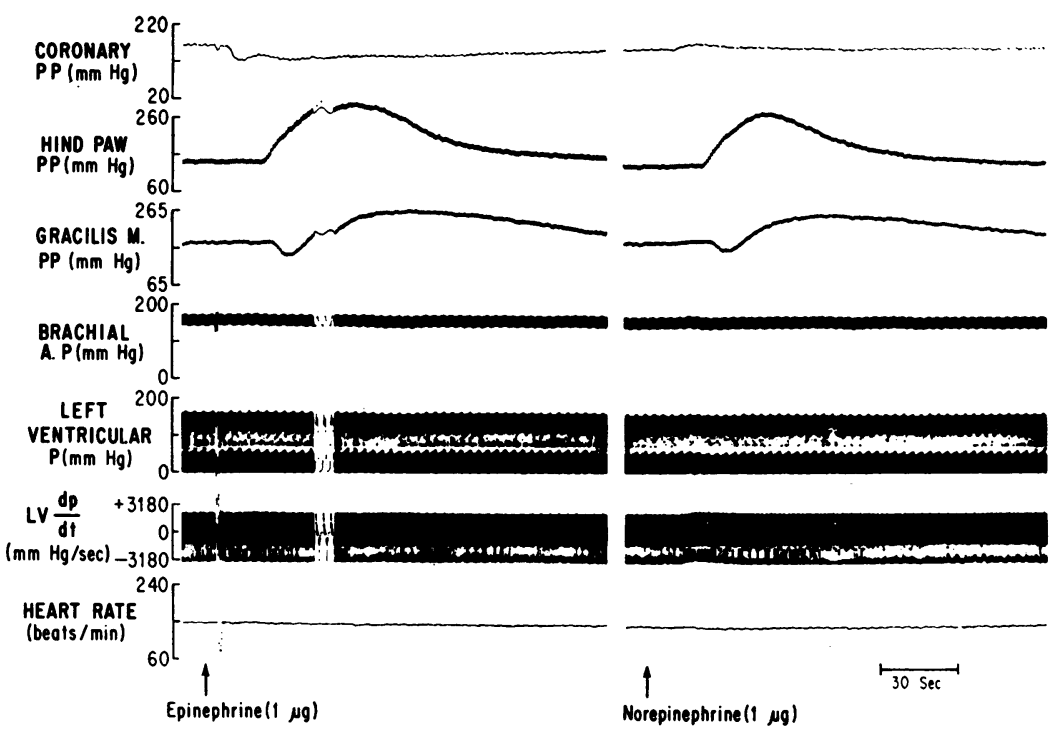

Figure 3 Responses to epinephrine and norepinephrine after practolol, $2 \mathrm{mg} /$ $\mathrm{kg}$ intravenously, in one of these experiments. Subsequent administration of propranolol reversed the coronary response to epinephrine from dilatation to constriction, but did not augment the constriction with norepinephrine. The transient dilator response in the gracilis muscle occurred occasionally with both agents, but not with injection of the vehicle. It was not consistently reduced by propranolol. (Exp. No. 157 VAH)

coronary responses to sympathetic nerve stimulation (11).

Finally, we believe the results of these experiments relate to the place of coronary vascular beta receptors in the classification of subtypes of beta adrenergic receptors. The pharmacological differentiation of beta receptors into at least two subtypes, beta-1 in myocardium and beta- 2 in vascular smooth muscle, has been based on differences in the relative potency of agonists and demonstration of selective beta receptor antagonists (17, 18). Two observations in the present study suggest that beta receptors in coronary vessels resemble those in peripheral vessels more than those in myocardium. First, the relative order of potency of agonists in activating coronary vascular beta receptors (isoproterenol $>$ epinephrine $>$ norepinephrine) resembled the order for peripheral vessels (isoproterenol $>$ epinephrine $>$ norepinephrine) and not the myocardium (isoproterenol $>$ norepinephrine $>$ epinephrine) (18). Second, practolol in the doses employed blocked myocardial but not coronary vascular responses to stimulation of beta receptors with isoproterenol. Subsequent administration of propranolol effectively antagonized coronary dilator responses to isoproterenol. These results are consistent with reports by three other groups of investigators that practolol in similar doses blocks myocardial but not coronary vascular beta receptors (19-21). In contrast, Lucchesi and Hodgeman (22) reported recently that practolol blocks coronary as well as myocardial beta receptors and suggested that beta receptors in coronary vessels and myocardium represent beta- 1 receptors while those in peripheral vessels represent beta- 2 receptors. These investigators found that $0.5 \mathrm{mg} / \mathrm{kg}$ of practolol given intravenously reduced coronary dilator as well as myocardial responses to isoproterenol. The reduction in the coronary dilator response could have resulted either from blockade of coronary vascular beta receptors or from decreases in the indirect coronary dilator effects of isoproterenol caused by myocardial stimulation. Lucchesi and Hodgeman concluded that the latter was unlikely, because intracoronary administration of calcium chloride, which produced an inotropic response approximating that observed with isoproterenol, caused only slight coronary dilatation. These investigators implied that coronary responses to calcium chloride result only from indirect effects produced by myocardial stimulation and therefore that myocardial stimulation contributes minimally to coronary vasodilatation since calcium chloride produced only slight dilatation. On the basis of these observations, they suggest that decreases in myocardial responses to isoproterenol after practolol could not explain the decreases in coronary dilator responses and concluded that practolol blocked coronary vascular beta receptors. The validity of this approach is doubtful, because calcium chloride also causes direct constrictor effects on coronary vessels (23) which would 
reduce the dilator responses produced by the inotropic effects of this agent (24). We did not observe a significant reduction of the coronary dilator response to isoproterenol after $1 \mathrm{mg} / \mathrm{kg}$ of practolol in this study, but did observe a slight reduction in a previous study (11). We attributed the slight reduction to inhibition of the indirect dilator action resulting from myocardial stimulation and not to blockade of coronary beta receptors. If the latter had occurred, administration of a higher dose of practolol, $2 \mathrm{mg} / \mathrm{kg}$, would have been expected to produce greater blockade of coronary beta receptors and greater reduction of the dilator response to isoproterenol. This was not seen in the present study in which myocardial responses were reduced or abolished by $1 \mathrm{mg} / \mathrm{kg}$ of practolol, but coronary responses were not altered by 1 or $2 \mathrm{mg} / \mathrm{kg}$. We conclude from these and other studies (19-21) that practolol in low doses blocks myocardial but not coronary or peripheral vascular beta receptors. These results, combined with the data on relative potency of agonists in this study, strongly suggest that the beta receptors in coronary vessels resemble those in peripheral vessels more than those in myocardium.

\section{ACKNOWLEDGMENTS}

This study was supported by Research and Education Associateships from the Veterans Administration, and by Grants HE-02644 and HE-09835 and Research Career Program Awards HE-K4-28749 and HE-K3-17013 from the National Heart and Lung Institute.

\section{REFERENCES}

1. Abboud, F. M., A. L. Mark, J. G. Hackett, and D. R. McRaven. 1970. Effects of adrenergic stimuli on coronary, cutaneous and muscular vessels. Circulation. 42: 82.

2. Shipley, R. E., and D. E. Gregg. 1945. The cardiac response to stimulation of the stellate ganglia and cardiac nerves. Amer. J. Physiol. 143: 396.

3. Denison, A. B., Jr., S. Bardhanabaedya, and H. D. Green. 1956. Adrenergic drugs and blockade on coronary arterioles and myocardial contraction. Circ. Res. 4: 653 .

4. Berne, R. M. 1958. Effect of epinephrine and norepinephrine on coronary circulation. Circ. Res. 6: 644.

5. Hardin, R. A., J. B. Scott, and F. J. Haddy. 1961. Effect of epinephrine and norepinephrine on coronary vascular resistance in dogs. Amer. J. Physiol. 201: 276.

6. Berne, R. M., H. DeJeest, and M. N. Levy. 1965. Influence of the cardiac nerves on coronary resistance. Amer. J. Physiol. 208: 763.

7. Granata, L., R. A. Olsson, A. Huvos, and D. E. Gregg. 1965. Coronary inflow and oxygen usage following cardiac sympathetic nerve stimulation in unanesthetized dogs. Circ. Res. 16: 114.

8. Klocke, F. J., G. A. Kaiser, J. Ross, Jr., and E. Braunwald. 1965. An intrinsic adrenergic vasodilator mechanism in the coronary vascular bed of the dog. Circ. Res. 16: 376.

9. Zuberhuhler, R. C., and D. F. Bohr. 1965. Responses of coronary smooth muscle to catecholamines. Circ. Res. 16: 431 .

10. Feig1, E. O. 1967. Sympathetic control of coronary circulation. Circ Res. 20: 262.

11. McRaven, D. R., A. L. Mark, F. M. Abboud, and H. E. Mayer. 1971. Responses of coronary vessels to adrenergic stimuli. J. Clin. Invest. 50: 773.

12. Dunlop, D., and R. J. Shanks. 1968. Selective blockade of adrenoceptive beta receptors in the heart. Brit. J. Pharmacol. Chemother. 32: 201.

13. Häggendal, J. 1963. An improved method for fluorometric determination of small amounts of adrenaline and noradrenaline in plasma and tissues. Acta Physiol. Scand. 59: 242 .

14. Anton, A. H., and D. F. Sayre. 1962. A study of the factors effecting the aluminum oxide trihydroxyindole procedure for the analysis of catecholamines. J. Pharmacol. Exp. Ther. 138: 360.

15. Foster, R. W. 1966. The pharmacology of pressor drugs. Brit. J. Anaesth. 38: 690.

16. King, M. P., E. T. Angelakos, and I. Uzgiris. 1971. Innervation of the coronaries. Fed. Proc. 30: 613.

17. Lands, A. M., A. Arnold, J. P. McAuliff, F. P. Luduena, and P. G. Brown, Jr. 1967. Differentiation of receptor systems activated by sympathomimetic amines. Nature (London). 214: 597.

18. Furchgott, R. F. 1967. The pharmacological differentiation of adrenergic receptors. Ann. N. Y. Acad. Sci. 139: 553.

19. Barrett, A. N., A. F. Crowther, D. Dunlop, R. G. Shanks, and L. H. Smith. 1968. Cardio-selective beta blockade. Naunyn-Schmeiderbergs Arch. Pharmakol. Exp. Pathol. 259: 156.

20. Ross, G., and C. R. Jorgensen. 1970. Effects of a cardioselective beta-adrenergic blocking agent on the heart and coronary circulation. Cardiovasc. Res. 4: 148.

21. Bussman, W. D., M. Rauh, and H. P. Krayenduehl. 1970. Coronary and hemodynamic effects of myocardioselective beta-receptor blockade by I.C.I. 50172 in the closed-chest dog. Differentiation of coronary and myocardial beta receptors. Amer. Heart J. 79: 347.

22. Lucchesi, B. R., and R. J. Hodgeman. 1971. Effect of 4-(2-hydroxy-3-isopropylaminopropoxy) acetanilide (AY 21,011 ) on the myocardial and coronary vascular responses to adrenergic stimulation. J. Pharmacol. Exp. Ther. 176: 200.

23. Scott, J. B., E. D. Frolich, R. A. Hardin, and F. J. Haddy. 1961 . $\mathrm{Na}^{+}, \mathrm{K}^{+}, \mathrm{Ca}^{++}$, and $\mathrm{Mg}^{++}$action on coronary vascular resistance in the dog heart. Amer. J. Physiol. 201 : 1095.

24. Pitt, B., Y. Sugishita, and D. E. Gregg. 1969. Coronary hemodynamic effects of calcium in the unanesthetized dog. Amer. J. Physiol. 216: 1456. 